\title{
Molecular Excitation and Radiative Transfer: Current Results and Future Prospects
}

\author{
John H. Black \\ Onsala Space Observatory, Chalmers University of Technology \\ S-439 92 Onsala, Sweden
}

\begin{abstract}
The spectra of dilute molecular gases in astrophysics arise in material far out of thermodynamical equilibrium (non-LTE), often in inhomogeneous atmospheres where Doppler motions from kinematical gradients and turbulence are large compared with thermal motions. Thus line intensities contain information about density and temperature as well as abundances, and line profiles probe the structure of the atmosphere. However, the theoretical description of the atmosphere can be extremely complicated and can make enormous demands for molecular data. Improvements in angular resolution and the possibility to make sensitive spectroscopic measurements at mid-infrared and submillimeter wavelengths pose new challenges for theoretical models.
\end{abstract}

\section{Introduction}

Stellar clusters, stars, planets, and small solid bodies evidently form out of dilute gas and dust. Their compositions and structures are shaped by gravitational and magnetic forces, which are mediated by the transport of radiation, gas flows, and a variety of microscopic processes. Spectroscopy of interstellar matter allows us to learn about the origins and evolution of these structures. The analysis is complicated because the matter is so dilute that it is often in a state far out of thermodynamical equilibrium (non-LTE). On the other hand, the richness of the molecular spectra affords numerous diagnostic tools.

Recent advances in observation present new challenges for theory. The high angular resolution achieved with interferometers and with adaptive optics reveals significant variations in intensity (and inferred abundances) on small linear scales. Spectroscopy at infrared and sub-mm wavelengths probes regions where molecular lines are formed in the presence of a strong continuum: models must now be able to describe true, stratified atmospheres in which lines and continuum are treated in a self-consistent manner.

Because molecular excitation and radiative transfer often appear to be intricate but dull subjects, let us try to enliven their discussion with a few provocative observations.

1. Molecular abundances are not directly observable properties: theorists should be obliged to compute synthetic spectra for realistic model clouds in order to derive abundances by comparison with observed spectra. 
2. The abundances and excitations of molecules are strongly coupled when reactive processes (state-dependent formation, destruction, ortho/para interchange, etc.) are rapid compared with excitation processes.

3. The unfortunate absence of data on inelastic collision rates is not a good excuse for avoiding non-LTE descriptions of molecular excitation.

4. The large number of energy states populated in molecules like $\mathrm{CH}_{3} \mathrm{OH}$ should not be used as a reason to ignore most of them.

5. There are many cases where observed mm-wave lines of interstellar or circumstellar molecules may have excitation that is partly controlled by radiative processes in the infrared: this is not a bad dream best forgotten, but rather an interesting diagnostic tool.

In the recent past, we have usually been presented with two distinct kinds of spectra. At ultraviolet and visible wavelengths, interstellar and circumstellar matter show pure absorption spectra against continuous backgrounds that arise elsewhere. The photon energy $h \nu / k \gg T$, where $T$ represents either the kinetic temperature of the absorbing atmosphere or the typical excitation temperature of molecules in it. At $\mathrm{cm}$ and $\mathrm{mm}$ wavelengths, the dilute gas typically shows simple emission spectra on top of a weak continuum, whose brightness temperature is close to that of the cosmic background radiation $\left(T_{\text {cbr }}=2.7 \mathrm{~K}\right)$. Although $h \nu / k \sim T$, the continuum is usually external to the source. In contrast, recent observations of star-forming regions at infrared and submillimeter wavelengths exhibit a mixture of emission and absorption spectra in the presence of a strong continuum. When the excitation temperatures $T_{\text {ex }}$ are comparable to the brightness temperature of the continuum, the absorption may be reduced by stimulated emission and $T_{\text {ex }}$ may be high enough that the "absorbing" gas produces significant line emission. In such atmospheres even the basic character of the spectrum (absorption versus emission) depends sensitively on the physical conditions (e.g. density and kinetic temperature) and their gradients.

These effects are illustrated dramatically by spectra of $\mathrm{H}_{2} \mathrm{O}$ molecules in the Orion IRc2 source obtained with the Infrared Space Observatory (ISO): at wavelengths $\lambda>50 \mu \mathrm{m}$, the rotational lines are seen in emission (Harwit et al. 1998), while at $\lambda=25$ to $45 \mu \mathrm{m}$ the rotational lines show net absorption, but with obvious emission components when the line profiles are resolved (Wright et al. 2000). There is also a component of steam: water that is hot enough to produce emission lines in the $\nu_{2}=1-0$ vibrational band at $\lambda \approx 6.7 \mu \mathrm{m}$ (van Dishoeck et al. 1998; González-Alfonso et al. 1998). Figure 1 displays the midinfrared spectrum of Orion IRc2 in a form that emphasizes the strong coupling between $\mathrm{H}_{2} \mathrm{O}$ lines and the continuum.

The combination of large, multi-level molecular systems and line-formation in inhomogeneous non-LTE atmospheres demands sophisticated methods of radiative transfer. Progress is being made along two different lines: Monte Carlo methods seem well suited to situations where an accurate representation of complex source structure is the goal, while explicit iterative techniques (e.g. approximate $\Lambda$-operators) find application to problems where the complexity lies in the molecular spectra and in the excitation processes. 

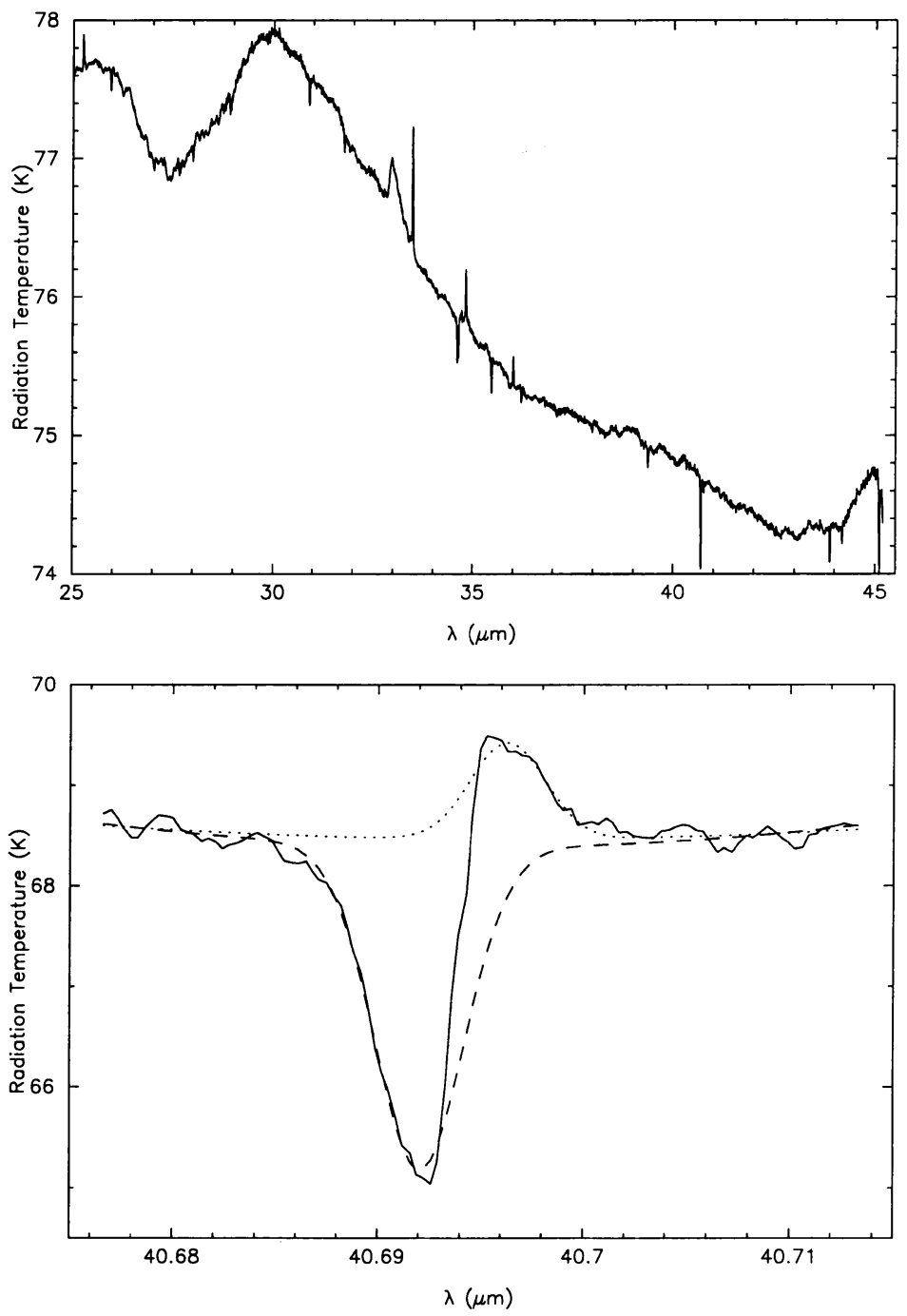

Figure 1. The ISO-SWS grating spectrum of Orion IRc2 adapted from Wright et al. (2000). The vertical scale is the Planckian radiation temperature corresponding to the observed flux in the aperture solid angle. Upper panel: Sharp absorption features are due to pure rotational lines of $\mathrm{H}_{2} \mathrm{O}$ with $E_{\ell} / k=200-750 \mathrm{~K}$. The sharp emission features are atomic nebular lines. The broad emission feature at 33 $\mu \mathrm{m}$ is an instrumental artefact. Lower panel: Profile of the $4_{32}-3_{03}$ line at a resolution of $10 \mathrm{~km} \mathrm{~s}^{-1}$ with the Fabry-Perot. The observed absorption arises from a small $(\approx 1 \mathrm{~K}$ or less) difference between the excitation temperature of $\mathrm{H}_{2} \mathrm{O}$ and the radiation temperature of the continuum. 
Conventional modelling in molecular astrophysics has usually decoupled the problem of multi-level radiative transfer from the description of the chemistry (i.e. the formation and destruction processes). In general, this is not necessary; indeed, in cases where the rates of formation and destruction are comparable to those of excitation processes it is inadvisable.

\section{The Nature of Dilute Atmospheres}

The general requirement for the new generation of theoretical models is a real "atmosphere", which represents both lines and continuum in a consistent manner. The ultimate goal is to perform large multi-level radiative transfer calculations in three dimensions with arbitrary distributions of density, temperature and abundances. Along with this, it is necessary to do a full spectrum synthesis in order to identify weak lines in confusion-limited spectra and to disentangle effects of abundance and excitation on the intensities of strong lines. It is instructive to contrast our dilute atmospheres with normal stellar atmospheres. In the dilute case, the scale of physical gradients is large and often resolvable. Departures from LTE are larger and more common, affect both chemical abundances and excitation, and can give rise to masers and lasers in extreme cases. The simple geometries of stellar atmospheres (plane parallel or spherical) can often be bad first approximations.

Molecular excitation can be strongly coupled to several different parts of the electromagnetic spectrum and to several different collision partners. Sources of continuous opacity are varied. In X-rays, inner-shell absorption by atoms (free atoms, atoms in molecules and atoms bound in dust grains) are important. In the ultraviolet, continuous absorption by atoms and molecules occurs, but may be less important as an opacity source than the absorption and scattering by dust. The non-zero albedo of dust leads to angle-dependent scattering, which introduces a further nasty complication to the radiative transfer. At wavelengths $\lambda=80-110 \mathrm{~nm}$, there is also a quasi-continuum formed by the strong resonance lines of $\mathrm{H}, \mathrm{H}_{2}$, and $\mathrm{CO}$. This wavelength range is especially important because the main processes of photoionization of $\mathrm{H}$ and $\mathrm{C}$ and photodissociation of $\mathrm{H}_{2}$ and $\mathrm{CO}$ compete for these photons. In the visible and infrared the opacity is dominated by dust and large molecules. At $\lambda>20 \mu \mathrm{m}$, the continuous emission comes primarily from thermal emission by warm dust. At shorter wavelengths in the infrared, there may be additional contributions from small dust particles undergoing temperature fluctuations and from the quasi-continua of the large molecules (e.g. PAHs) thought to be responsible for the so-called unidentified IR (UIR) emission bands (see Giard et al. 1997). In the far-infrared and submillimeter regions, even cold dust particles $\left(T_{\text {dust }} \approx 15 \mathrm{~K}\right)$ produce measurable emission. At submm and short $\mathrm{mm}$ wavelengths the forest of molecular lines accounts for a significant fraction of broad-band flux.

The profiles of molecular lines are affected by several broadening mechanisms including thermal Doppler motions, some form of "turbulence", and systematic large-scale motions such as rotation, collapse, expansion, and shock waves. Although collisional broadening is important in stellar and planetary atmospheres and for some atomic lines in the densest ionized nebulae, there are no known instances in dilute molecular gas. Line shapes can be affected by co- 
herent effects in masers. Although the conventional wisdom suggests that the microscopic speed distributions of electrons, atoms, and molecules will be well thermalized and thus Maxwellian even at low interstellar densities (e.g. Spitzer 1978 , chapter 2), it is possible that non-Maxwellian excesses in some species will need more attention in the future.

\section{Collision Cross Sections and Rates}

It is through collisions with atoms, electrons, and other molecules that the signatures of physical variables (density, temperature, etc.) are written into the spectra of dilute molecular atmospheres. These processes also partly control the physical state and evolution of the system, especially through cooling. A molecule cools the gas as energy is transferred from kinetic motions into radiation that escapes. Formally the rate of cooling by a molecule is

$$
L=\sum_{\ell} \sum_{u} \sum_{c} \Delta E_{u \ell} n_{c}\left(n_{\ell} q_{\ell u}^{c}-n_{u} q_{u \ell}^{c}\right) \quad \operatorname{ergs~} \mathrm{cm}^{-3} \mathrm{~s}^{-1}
$$

where $q_{u \ell}^{c}$ is the rate coefficient of collision-induced transitions between upper and lower states $u$ and $\ell$ that are separated by energy $\Delta E_{u \ell}$ and $n_{c}$ is the number density of collision partner $c$. The densities $n_{\ell}$ and $n_{u}$ depend non-linearly on the non-local radiative transfer in a multi-level system: that is, the cooling at any point in the atmosphere depends upon whether a photon created there actually escapes (cools) or not. It is sometimes mistakenly claimed that the observed power in emission lines measures directly the cooling: this is true only to the extent that the observed power derives ultimately from collisions. In cases where molecular excitation is dominated by radiation that does not derive from inelastic collisions, the observed power can differ from the true cooling. For example, in the interstellar medium the dust absorbs and re-radiates approximately 100 times as much power as the gas. The dust and gas are in intimate thermal contact with each other only at rather high gas densities. Thus molecules like $\mathrm{H}_{2} \mathrm{O}$ that couple strongly to the IR/submm continuum radiation may be excited to radiate without extracting much kinetic energy from the gas. This effect was already pointed out by Takahashi, Silk, \& Hollenbach (1983). Another example is the infrared emission in quadrupole vibrational and rotational transitions of $\mathrm{H}_{2}$, which can be excited by ultraviolet absorption and fluorescence (e.g. Black \& van Dishoeck 1987; Sternberg \& Dalgarno 1989).

The cross sections (or corresponding thermal rate coefficients) for inelastic collisions with $\mathrm{H}_{2}$ that excite rotation and vibration are known for only a few astrophysically interesting molecules. These cross sections are rarely measured. Although accurate quantum mechanical computations are possible, they require very accurate potential energy surfaces, which in turn require labor-intensive computations. Complex molecules like $\mathrm{CH}_{3} \mathrm{OH}$ exhibit hundreds of measurable lines in the spectra of interstellar clouds: the excitation of these lines is controlled in part by thousands of collisional processes. The transitions of interest involve not only rotational levels of the ground vibrational state but also the corresponding levels of excited torsional modes. Even in fully molecular regions where $\mathrm{H}_{2}$ is expected to be the dominant collision partner, the cross sections can be quite different for $\mathrm{H}_{2}$ in its para ground state $J=0$ and in its ortho state 
$J=1$. In some instances, $\mathrm{H}$ and $\mathrm{He}$ can be important collision partners, and electron-impact excitation of polar or ionic species must be considered when the fractional ionization exceeds $n(e) / n\left(\mathrm{H}_{2}\right) \approx 10^{-5}$. There are some reactive collisions that affect the excitation of molecules. In particular, reactive collisions with $\mathrm{H}, \mathrm{H}^{+}$and $\mathrm{H}_{3}^{+}$are effective in changing the nuclear-spin species (ortho and para forms) of molecules like $\mathrm{H}_{2}, \mathrm{H}_{2} \mathrm{CO}$, and $\mathrm{H}_{2} \mathrm{O}$. Ortho/para ratios are often assumed to be unchangeable and thus to derive solely from formation processes; on the contrary, they should be considered as results of the competition between spin-changing reactive processes on the one hand and the processes of formation, destruction, and excitation on the other.

The lack of accurate inelastic collision rates for many astrophysically important molecules is a serious problem. Densities cannot be derived from observed intensities without them, nor can collisionally pumped masers be correctly explained. As indicated above (eq. 1) the true cooling rates cannot be evaluated reliably without knowledge of the collision rates. One might hope that in limiting cases the exact values of collision cross sections may not be crucial: when the most populous, observed levels of a molecule are clearly well thermalized by collisions, the details are less important; and when the excitation is completely dominated by radiative processes, the collision cross sections and densities would seem to be less important than a realistic treatment of the radiative transfer.

In the absence of detailed knowledge, one can estimate collision rate coefficients that are simply scaled to the radiative line strengths for allowed transitions: in fact, this is a bad assumption for many molecules in which the most likely collision-induced transitions will not follow dipole selection rules, but it permits a crude assessment of the level of competition between radiative and collisional processes. It is also possible to adopt cross sections that have been determined accurately for some other molecule that has the same kind of electronic state and similar values of rotational constants and dipole moments. At the very least, educated guesses should make it possible to identify those systems for which the lack of accurate data is most crucial. Because the ab initio computation of collision cross sections is tedious and somewhat unappreciated (perjoratively "measurement" rather than experiment), it is important to encourage and support colleagues who measure or calculate them!

Two examples of recent work on collisional excitation of small molecules deserve special emphasis. First, the excitation of $\mathrm{H}_{2}$ by $\mathrm{H}, \mathrm{H}_{2}$, and $\mathrm{He}$ continues to be studied (Flower 1998; Balakrishnan et al. 1999; Flower, Roueff, \& Zeippen 1998). The $\mathrm{H}-\mathrm{H}_{2}$ collision system is especially important in hot, shocked regions of the interstellar medium and in the cooling properties of primordial gas; accordingly, much effort has gone into computing accurate cross sections for vibrational as well as rotational transitions involving highly excited states over a large range of collision energies (Mandy \& Martin 1993; Martin \& Mandy 1995; Martin, Schwarz, \& Mandy 1996; Sun \& Dalgarno 1994; Lepp, Buch, \& Dalgarno 1995; Forrey et al. 1997; Flower 1997; Flower \& Roueff 1998; Le Bourlot, Pineau des Forêts, \& Flower 1999). The results are often quite sensitive to the adopted interaction potential (Lepp et al. 1995) and these potential surfaces have continued to evolve. Corresponding collision-induced transitions in HD have been studied (Roueff \& Flower 1999). It is important to recognize that reactive, $\mathrm{H}$-exchange processes can occur and that these have large rate 
coefficients $\left(\approx 10^{-11} \mathrm{~cm}^{3} \mathrm{~s}^{-1}\right)$ for highly excited $\mathrm{H}_{2}$ and for the lowest states at high temperatures $(T \geq 300 \mathrm{~K})$. Since the reactive scattering occurs mainly at small inter-nuclear distances where the interaction potential is best determined, the existing rates (Sun \& Dalgarno 1994) are rather insensitive to differences in potential surfaces. Because the orientation of nuclear spin can change in such a reactive process, this is a mechanism for ortho/para interchange

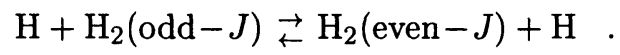

Moreover, the analogous deuterium atom exchange reaction

$$
\mathrm{D}+\mathrm{H}_{2} \underset{\mathrm{HD}}{\rightleftarrows}+\mathrm{H}
$$

might be able to couple the rotational and vibrational excitation of HD more closely to that of $\mathrm{H}_{2}$ than non-reactive inelastic collisions with $\mathrm{H}_{2}$ alone, in a warm photon-dominated region (PDR) or shock where the atomic fraction is relatively high $\left(\mathrm{H} / \mathrm{H}_{2} \precsim 0.01\right)$.

Second, there has been a mildly surprising development in our understanding of rotational excitation of small molecules by electron impact. Under previous simplifying assumptions, it has been suggested that dipole selection rules (i.e. change by \pm 1 rotational quantum) will be strongly favored in collisions between electrons and molecular ions or polar neutrals (Dickinson \& Flower 1981; Neufeld \& Dalgarno 1989). More sophisticated quantum mechanical treatments of electron-impact excitation of $\mathrm{NO}^{+}, \mathrm{HeH}^{+}$(Rabadán, Sarpal, \& Tennyson 1998) and $\mathrm{CH}^{+}$(Lim, Rabadán, \& Tennyson 1999) show that transitions of two or more rotational quanta are comparable or even favored. Electron energy deposition in a gas of $\mathrm{H}, \mathrm{H}_{2}$, and $\mathrm{He}$ has recently been reviewed (Dalgarno, Yan, \& Liu 1999) and new calculations of $e$-impact dissociation of $\mathrm{H}_{2}$ have been performed (Stibbe \& Tennyson 1999).

\section{Methods of Radiative Transfer}

How is it possible to treat inhomogeneous, non-LTE atmospheres in the detail demanded by current and anticipated observations? There are at least two different kinds of problems of interest. One is to represent realistically the effect of all scales of internal structure of the inhomogeneous atmosphere upon the emission lines of a few simple molecules in order to extract the full physical meaning from maps of varying brightness and Doppler velocity. The other is to interpret fully the crowded spectra of complex species in order to derive accurate abundances and physical conditions (e.g. density, temperature, ionization rate and so on) from a number of line profiles. Clearly, it is not now possible to simulate the entire spectrum of an interstellar cloud from $100 \mathrm{~nm}$ to $100 \mathrm{~cm}$ wavelength including thousands of transitions each of the few hundred most important molecules plus two or three components of dust. However, considerable progress has already been made in simulating the spectra of turbulent clouds.

Let us summarize first the basic theoretical problem, in order to identify crucial assumptions. In simplest terms, there are two sets of equations that must 
be solved simultaneously. The kinetic equations

$$
\frac{d}{d t} n_{i}(t)=F_{i}-n_{i} D_{i}+n_{j} \sum_{j \neq i} P_{j i}-n_{i} \sum_{j \neq i} P_{i j}
$$

describe the number density $n_{i}$ of a molecule in state $i$. Usually this is reduced to statistical equilibrium in steady state by setting $d n_{i}(t) / d t=0$. The kinetic equations are local, that is they apply to the concentration in each state at a single point in the atmosphere. The radiative transfer equation

$$
\frac{d I_{\nu}}{d \tau_{\nu}}=-I_{\nu}+S_{\nu}
$$

on the other hand is non-local: that is, the intensity $I_{\nu}$ at each point in space and at frequency $\nu$ has contributions not only from the local source function $S_{\nu}$, but also from radiating molecules elsewhere in the atmosphere. The rate elements in the kinetic equations $P_{i j}=C_{i j}+R_{i j}$ include both collisional $\left(C_{i j}\right)$ and radiative $\left(R_{i j}\right)$ terms: the former are local, but the latter depend on the intensity at each point averaged over all directions within the atmosphere and integrated over the line profile. It has been common in the past to ignore the formation and destruction terms, $F_{i}$ and $n_{i} D_{i}$, respectively, in the kinetic equations. In that case, an additional normalization equation is introduced and the system is solved for the fractional populations. The importance of including the sources and sinks explicitly is discussed in detail elsewhere (Lindholm \& Black 2000). Formation of a molecule by exoergic processes can provide a significant input directly into its excited states. The effects of such processes will be most noticeable when the destruction rates approach the rates of normal excitation processes. Specifically, there is a class of highly reactive molecules, such as $\mathrm{CH}^{+}$and $\mathrm{CO}^{+}$, which react on almost every collision with the principal neutrals $\mathrm{H}$ and $\mathrm{H}_{2}$ : it is unlikely that the rotational populations of such species can ever be fully thermalized in neutral interstellar gas (Black 1998). In summary, the kinetic equations and the transfer equation are coupled to each other in a non-linear way. Their simultaneous solution becomes even more complicated when large numbers of molecular states and transitions are involved and when the range of optical depths is large.

There is a hierarchy of approximate methods that can be applied to the solution of the coupled equations.

1. The assumption of a uniform excitation temperature for all states eliminates the need to solve the kinetic equations. In one of the limiting cases of optical depth, $\tau_{i j} \ll 1$ or $\tau_{i j} \gg 1$, the transfer equation also simplifies.

2. The rotational diagram method also presumes a uniform excitation temperature, but allows its value to be derived (rather than assumed) from data on several transitions of the same molecule. It is also possible to incorporate corrections for the optical depth in each line. An illuminating discussion of this method has appeared recently (Goldsmith \& Langer 1999).

3. A generalized curve of growth can be derived, in which the effects of stimulated emission and strong continuum coupling are included. The curve of 
growth naturally treats line saturation. Thus it can supplant analysis by the rotation diagram method with only slightly more effort. The results of a curve-of-growth analysis can serve as a first approximation solution in an iterative, explicit radiative transfer model.

4. Radiative transfer in uniform or systematically varying atmospheres can be treated, for example, by mean escape probabilities or the large velocity gradient (Sobolev) method (Sobolev 1960). Computed line profiles contain information only about the assumptions that were built into the model. An empirical continuum can easily be included. It is practical to explore the excitation and line intensities of molecules with a very large number of transitions. These methods in their simplest forms may be inadequate for transitions of extremely high optical depth. The simple methods can identify population inversions and some maser transitions.

5. Explicit methods of solving the multi-level, non-LTE, radiative transfer equation exist. Rapid progress is being made by many groups in applying several techniques to the inhomogeneous atmospheres of arbitrary geometry that are of interest to us. A brief comparison will be made of Monte Carlo methods and Lambda-Iteration methods (§4.2).

Finally, a specialized workshop on "Radiative Transfer in Molecular Lines" convened in Leiden 1999 May 17-21 to discuss the advanced methods. Useful resources for radiative transfer have been assembled from that workshop and are available through http://www.strw. leidenuniv.nl/ radtrans.

\subsection{Generalized curve of growth}

Interstellar absorption lines in the visible and ultraviolet are typically cases of pure absorption in which the absorbing gas is physically separate from the background continuum source and is too cold $\left(T_{\text {ex }} \ll h \nu / k\right)$ to produce emission at the line frequencies. In that case, the classical equivalent width

$$
W_{\nu}=\int \frac{I_{c}-I_{\nu}}{I_{c}} d \nu=\int\left(1-e^{-\tau(\nu)}\right) d \nu
$$

gives the integrated line intensity in relation to the brightness of the continuum $\left(I_{c}\right)$ and is a function of the lower-state column density only, with a parametric dependence on the Doppler line width. In the infrared and submillimeter spectra of star-forming molecular clouds and circumstellar envelopes, we must deal with other complications: the molecular excitation can be hot with respect to the photon energy $\left(T_{\mathrm{ex}} \sim h \nu / k\right)$ so that an absorbing layer can also produce significant emission, and stimulated emission may be important. In addition, the continuum source may be extended compared with the size of structures in the foreground gas and it may be near (or part of) the line-forming region. Thus the observed intensity at the line frequency

$$
I_{\mathrm{obs}}(\nu)=(1-a) I_{c}(\nu)+a I_{c}(\nu) e^{-\tau_{u \ell}(\nu)}+b B_{\nu}\left(T_{u \ell}\right)\left(1-e^{-\tau_{u \ell}(\nu)}\right)
$$

where $B_{\nu}\left(T_{u \ell}\right)$ is the Planck function at the excitation temperature $T_{u \ell}$ of the transition $u \rightleftarrows \ell, a$ is the fractional area of the continuum covered by the absorber, 
and $b$ is a filling factor of emitting gas. The optical depth $\tau_{u \ell}(\nu)$ now includes the correction for stimulated emission. The generalized equivalent width is

$$
W_{\nu}^{\mathrm{obs}}=\int\left[\left(a-b \frac{B_{\nu}\left(T_{u \ell}\right)}{I_{c}(\nu)}\right)\left(1-e^{-\tau_{u \ell}(\nu)}\right)\right] d \nu
$$

and the classical formula is recovered for a fully covered point-like continuum source, $a=1$, and in the limit of weak emission, $b B_{\nu}\left(T_{u \ell}\right) / I_{c}(\nu) \sim 0$. This simple approach incorporates several important effects in a transparent way: the competition between emission and absorption and competition between lines and continuum can be included with an empirical description of the continuous spectrum. The formula is also easily adaptable to non-linear optimization in fitting data. It is important to note that the integrated intensity of each line now depends on the column densities in both the upper and lower states with parametric dependencies on the Doppler width and the geometrical factors. It is also clear that when the continuum is strong and the molecules strongly coupled to it, it is important to measure the continuum brightness at the same time as the line intensity. This method is no substitute for a true model atmosphere, but it can be very useful when several different transitions of the same molecule are observed. It has been applied successfully in an analysis of the ISO-SWS spectrum of Orion IRc2 in which 19 rotational lines of $\mathrm{H}_{2}$ appear predominantly in absorption at 25-45 $\mu \mathrm{m}$ (Wright et al. 2000). The analysis shows that these high-excitation lines are excited mainly by the strong continuum with a mean $T_{\text {ex }} \approx 72 \mathrm{~K}$ and that the derived total column density $N\left(\mathrm{H}_{2} \mathrm{O}\right)=1.5 \times 10^{18} \mathrm{~cm}^{-2}$ is rather insensitive to the values of the geometrical factors. Moreover, the simple analysis correctly reproduces the relative amounts of emission and absorption in the line profiles that have been observed at high resolution (see Figure 1). Details of the method and exemplary applications will be discussed elsewhere (Black 2000).

\subsection{Small-scale structure in molecular clouds and young stellar ob- jects}

The radiating surfaces of quiescent molecular clouds show small-scale $(0.01 \mathrm{pc}$ or less) fluctuations both in brightness and in Doppler velocity (e.g. Falgarone et al. 1998). Various approaches have been developed to analyze such structure (Scalo 1984; Falgarone \& Phillips 1990; Houlahan \& Scalo 1990, 1992; Lis et al. 1996; Heyer \& Schloerb 1997; Stutzki et al. 1998; Rosolowsky et al. 1999), which is inferred to be a manifestation of interstellar turbulence. Parallel developments in numerical simulations of turbulent gas clouds now require elaborate methods of radiative transfer in order to draw connections between the distribution of physical variables (density, temperature, abundance, and velocity) and the observable distribution of emission line intensities and profile shapes. The initial comparisons of synthetic molecular clouds with real ones (e.g. Falgarone et al. 1994) have evolved into computationally demanding radiative transfer models (Juvela 1997; Padoan et al. 1998; Rousseau, Chaté, \& Le Bourlot 1998; Ossenkopf et al. 1999). So far, such radiative transfer calculations have been done for molecules like CO and CS, for which the excitation is simple (collisions in the cosmic background radiation), but where the lines form in a very inhomogeneous 
medium with no geometrical symmetry. Probabilistic methods (i.e. Monte Carlo simulations) are well suited to such problems.

Monte Carlo methods have been applied to multi-level radiative transfer in molecular clouds for some time (Bernes 1979), and in recent years have been extended from spherically symmetric clouds (e.g. Choi et al. 1995) to fully threedimensional cloud models (e.g. Park \& Hong 1995, 1998; Park, Hong, \& Minh 1996). They have also been applied to some masers (Spaans \& van Langevelde 1992 ) and to the transfer of radiation in the ultraviolet lines that both destroy and excite $\mathrm{H}_{2}$ in diffuse molecular clouds (Spaans \& Neufeld 1997). Monte Carlo methods have prodigious appetites for computing time; however, their efficiency can be greatly enhanced through iterative adjustment of the reference radiation field and other improvements (e.g. Juvela 1997).

As the resolution of radio-frequency images improves through sensitive interferometry, more complex structure appears in molecular line sources such as young stellar objects (YSOs). Such sources are often quite complicated, with contributions from associated static clouds, collapsing envelopes, surfaceirradiated disks, and outflows, as well as the extended atmosphere of the YSO per se. Monte Carlo simulations of multi-level transfer in two and three dimensions have already been applied to the interpretation of interferometric observations of such sources (e.g. Hogerheijde 1998; Hogerheijde et al. 1999; Kemper et al. 1999).

\subsection{Molecular clouds and masers}

In situations where lines and continuum must be treated together and where the spectra themselves are complex, other techniques in radiative transfer can be valuable. Explicit iterative calculations based on approximate lambda operators have been widely applied to stellar atmospheres (e.g. Scharmer 1984; Scharmer \& Carlsson 1985) and have great promise for interstellar applications. Accelerated lambda iteration (ALI) methods have been discussed by Rybicki \& Hummer $(1991,1992)$ and by Hubeny \& Lanz (1992). Techniques for improving the performance of ALI calculations continue to be developed (e.g. Atanacković-Vukmanović et al. 1997; Socas-Navarro \& Trujillo Bueno 1997). Doty \& Neufeld (1997) have incorporated ALI methods in models of dense molecular clouds. These methods have also been applied to $\mathrm{OH}$ lines in Galactic starforming regions (Jones et al. 1994) and in extragalactic mega-maser sources (Randell et al. 1995) and to $\mathrm{H}_{2} \mathrm{O}$ masers (Yates, Field, \& Gray 1997).

Acknowledgments. The Chalmers Foundation has provided generous support of this research. Additional support for investigations with ISO has been provided by NASA through a grant to the University of Arizona.

\section{References}

Atanacković-Vukmanović, O., Crivellari, L., \& Simonneau, E. 1997, ApJ, 487, 735

Balakrishnan, N., Forrey, R.C., \& Dalgarno, A. 1999, ApJ, 514, 520

Bernes, C. 1979, A\&A, 73, 67

Black, J.H. 1998, Faraday Disc., 109, 257

2000 , in preparation 
Black, J.H. \& van Dishoeck, E.F. 1987, ApJ, 322, 412

Choi, M., Evans, N.J. II, Gregersen, E.M., \& Wang, Y. 1995, ApJ, 448, 742

Dalgarno, A., Yan, M., \& Liu, W. 1999, ApJS, 125, 237

Dickinson, A.S. \& Flower, D.R. 1981, MNRAS, 196, 297

Doty, S.D. \& Neufeld, D. 1997, ApJ, 489, 122

Falgarone, E., Lis, D.C., Phillips, T.G., Pouquet, A., Porter, D.H., \& Woodward, P.R. 1994, ApJ, 436, 728

Falgarone, E. \& Phillips, T.G. 1990, ApJ, 359, 344

Falgarone, E., Panis, J.-F., Heithausen, A., Perault, M., Stutzki, J., Puget, J.-L., \& Bensch, F. 1998, A\&A, 331, 669

Flower, D.R. 1997, MNRAS, 288, 627 1998, MNRAS, 297, 334

Flower, D.R. \& Roueff, E. 1998, J. Phys. B, 31, L955

Flower, D.R., Roueff, E., \& Zeippen, C.J. 1998, J. Phys. B, 31, 1105

Forrey, R.C., Balakrishnan, N., Dalgarno, A., \& Lepp, S. 1997, ApJ, 489, 1000

Giard, M., Puget, J.L., Créte, E., \& Scoupe, F. 1997, A\&A, 322, 624

Goldsmith, P.F. \& Langer, W.D. 1999, ApJ, 517, 209

González-Alfonso, E., Cernicharo, J., van Dishoeck, E.F., Wright, C.M., \& Heras, A. 1998, ApJ, 502, L169

Harwit, M., Neufeld, D.A., Melnick, G.J., \& Kaufman, M.J. 1998, ApJ, 497, L105

Heyer, M.H. \& Schloerb, F.P. 1997, ApJ, 475, 173

Hogerheijde, M. 1998, PhD Thesis, Leiden

Hogerheijde, M., van Dishoeck, E.F., Salverda, J.M., \& Blake, G.A. 1999, ApJ, 513,350

Houlahan, P. \& Scalo, J. 1990, ApJS, 72, 133 1992, ApJ, 393, 172

Hubeny, I., \& Lanz, T. 1992, A\&A, 262, 501

Jones, K.N., Field, D., Gray, M.D., \& Walker, R.N.F. 1994, A\&A, 288, 581

Juvela, M. 1997, A\&A, 322, 943

Kemper, C., Spaans, M., Jansen, D.J., Hogerheijde, M., van Dishoeck, E.F., \& Tielens, A.G.G.M. 1999, ApJ, 515, 649

Le Bourlot, J., Pineau des Forêts, G., \& Flower, D.R. 1999, MNRAS, 305, 802

Lepp, S., Buch, V., \& Dalgarno, A. 1995, ApJS, 98, 345

Lim, A.J., Rabadán, I., \& Tennyson, J. 1999, MNRAS, 306, 473

Lindholm, J. \& Black, J.H. 2000, in preparation

Lis, D.C., Pety, J., Phillips, T.G., \& Falgarone, E. 1996, ApJ, 463, 623

Mandy, M.E. \& Martin, P.G. 1993, ApJS, 86, 199

Martin, P.G. \& Mandy, M.E. 1995, ApJ, 455, L89

Martin, P.G., Schwarz, D.H., \& Mandy, M.E. 1996, ApJ, 461, 265

Neufeld, D. \& Dalgarno, A. 1989, Phys. Rev. A, 40, 633

Ossenkopf, V., Bensch, F., MacLow, M.-M., \& Stutzki, J. 1999, in The Physics and Chemistry of the Interstellar Medium (Proceedings of the 3rd CologneZermatt Symposium), eds. V. Ossenkopf, J. Stutzki, \& G. Winnewisser, GCA-Verlag

Padoan, P., et al. 1998, ApJ, 504, 300

Park, Y.-S. \& Hong, S.S. 1995, A\&A, 300, 890 1998, ApJ, 494, 605

Park, Y.-S., Hong, S.S., \& Minh, Y.C. 1996, A\&A, 312, 981

Rabadán, I., Sarpal, B.K., \& Tennyson, J. 1998, J. Phys. B, 31, 2077 
Randell, J., Field, D., Jones, K.N., Yates, J.A., \& Gray, M.D. 1995, A\&A, 300, 659

Roueff, E. \& Flower, D.R. 1999, MNRAS, 305, 353

Rousseau, G., Chaté, H., \& Le Bourlot, J. 1998, MNRAS, 294, 373

Rosolowsky, E.W., Goodman, A.A., Wilner, D.J., \& Williams, J.P. 1999, ApJ, 524,887

Rybicki, G.B. \& Hummer, D.G. 1991, A\&A, 245, 171 $1992, \mathrm{~A} \& \mathrm{~A}, 262,209$

Scalo, J.M. 1984, ApJ, 277, 556

Scharmer, G.B. 1984, in Methods in Radiative Transfer, ed. W. Kalkofen, (Cambridge: Cambridge Univ. Press), 173

Scharmer, G.B. \& Carlsson, M. 1985, J. Comp. Phys., 59, 56

Sobolev, V.V. 1960, Moving Envelopes of Stars, (Cambridge: Harvard U. Press)

Socas-Navarro, H. \& Trujillo Bueno, J. 1997, ApJ, 490, 383

Spaans, M. \& Neufeld, D.A. 1997, ApJ, 484, 785

Spaans, M. \& van Langevelde, H.J. 1992, MNRAS, 307, 271

Spitzer, L.Jr. 1978, Physical Processes in the Interstellar Medium, (New York: Wiley)

Sternberg, A. \& Dalgarno, A. 1989, ApJ, 338, 197

Stibbe, D. \& Tennyson, J. 1999, ApJ, 513, L147

Stutzki, J., Bensch, F., Heithausen, A., Ossenkopf, V., \& Zielinsky, M. 1998, A\&A, 336, 697

Sun, Y. \& Dalgarno, A. 1994, ApJ, 427, 1053

Takahasi, T., Silk, J., \& Hollenbach, D.J. 1983, ApJ, 275, 145

van Dishoeck, E.F., Wright, C.M., Cernicharo, J., González-Alfonso, E., de Graauw, Th., Helmich, F.P., \& Vandenbussche, B. 1998, ApJ, 502, L173

Wright, C.M., van Dishoeck, E.F., Black, J.H., Feuchtgruber, H., Cernicharo, J., González-Alfonso, E., \& de Graauw, Th. 2000, A\&A, in press

Yates, J.A., Field, D., \& Gray, M.D. 1997, MNRAS, 285, 303 


\section{Discussion}

D. A. Williams: Under what conditions will $\mathrm{H}_{3}^{+}$be an important collision partner, and what are the prospects of making accurate cross section calculations for such processes?

J. Black: $\mathrm{H}_{3}^{+}$is thought to be important in ortho/para interchange through reactive proton-exchange collisions. Recent observations of interstellar $\mathrm{H}_{3}^{+}$itself show equilibrated ortho/para populations, attesting to the efficiency of such processes. The process has been discussed by $\mathrm{T}$. Oka and collaborators.

E. Herbst: Is the chemical destruction of newly formed $\mathrm{H}_{2} \mathrm{D}^{+}$competitive with de-excitation?

J. Black: The calculations for $\mathrm{H}_{2} \mathrm{D}^{+}$have not been performed yet.

M. Walmsley: Your "generalized" curve of growth must depend sensitively on geometrical factors (covering fractions etc.). Could you explain on how you deal with this?

J. Black: The geometrical factors can sometimes be constrained by other observations, e.g. high-resolution maps of source structure in related lines or continuum. In the case of $\mathrm{H}_{2} \mathrm{O}$ absorption toward Orion IRc 2, the derived total column density was insensitive to the geometrical factors. 